\title{
Histerectomía extrafascial en cáncer de cuello uterino IB2 posterior a radio-quimioterapia
}

\author{
Catalina Alonso M. 1,3, Erasmo Bravo O. 1,2,3, Sergio Rojas S. 1,2,3, \\ Santiago Parry R. 2,3 \\ 1 Unidad de Ginecología Oncológica, Hospital Gustavo Fricke de Viña del Mar. ${ }^{2}$ Unidad de Ginecología Oncológica, \\ Hospital Carlos van Buren de Valparaíso. ${ }^{3}$ Departamento de Ginecología y Obstetricia, Universidad de Valparaíso, Chile.
}

\section{RESUMEN}

Objetivo: Evaluar la sobrevida global y libre de enfermedad en pacientes con tratamiento concomitante de radioquimioterapia más histerectomía extrafascial en cáncer de cuello uterino IB2. Método: 31 pacientes con cáncer cérvico-uterino en etapa IB2 fueron tratadas con radioquimioterapia concomitante más histerectomía extrafascial programada en 4 a 6 semanas. Se efectuó radioterapia pelviana externa e implante de braquiterapia útero-vaginal con dosis preoperatoria entre $70-75$ Gy a punto $A$ y quimioterapia con cisplatino. Resultados: La edad promedio de las pacientes fue de $41 \pm 8$ años. El $81 \%$ de los casos fue carcinoma escamocelular. La histerectomía extrafascial se realizó 4 a 6 semanas post-radiación en el $85 \%$ de los casos. El $79 \%$ y el $13 \%$ de las pacientes recibieron 5 y 4 ciclos de quimioterapia respectivamente. La mediana de seguimiento fue de 38 meses. La sobrevida global y libre de enfermedad estimada a 5 años fue de $86 \%$ y $79 \%$ respectivamente. Hubo diferencia significativa en la sobrevida global en los subgrupos sin o con enfermedad residual macroscópica en la pieza operatoria $(p<0,001)$. Conclusión: Esta serie muestra resultados de sobrevida similares a las reportadas en otros trabajos con el mismo tratamiento. La enfermedad residual en la pieza operatoria podría constituir un factor de valor pronóstico.

\section{PALABRAS CLAVE: Cáncer cervicouterino IB2, radioquimioterapia, histerectomía extrafascial}

\section{SUMMARY}

Aim: To evaluate overall and disease free survival in cervical cáncer IB2 patients with concomitant chemoradiotherapy and posterior extrafascial hysterectomy treatment. Methods: Between July 2005 to December 2009, a total of 31 eligible patients with IB2 cervical cancer were treated with chemoradiotherapy and posterior hysterectomy in the Carlos Van Buren Hospital Oncology Unit. Radiotherapy consisted in external pelvic radiation and a utero-vaginal brachitherapy with low rate doses to get preoperatory doses between 70 and 75 Gy to the A point and a concomitant Cisplatin based chemotherapy. After this treatment, the patients received extrafascial hysterectomy 4 to 6 weeks completed chemoradiotherapy. Results: The mean age was $41 \pm 8$ years. $81 \%$ of the patients had an spinocelular carcinoma. The extrafascial hysterectomy was made between 4 to 6 weeks post-radiation in $85 \%$ of the patients. $79 \%$ and $13 \%$ of the patients received 5 and 4 chemotherapy cycles respectively. The median follow up was 38 months. The 5 years overall survival and disease free survival estimates were $86 \%$ and $79 \%$ respectively. There was a significant difference between subgroups of patients with and without macroscopic residual disease in the operatory specimen $(p<0.001)$. 
Conclusion: Our survival is similar to published results with the same treatment. The presence of macroscopic residual disease in the hysterectomy specimen could be a factor of prognostic value.

KEY WORDS: Cervical carcinoma IB2, chemoradiotherapy, extrafascial hysterectomy

\section{INTRODUCCIÓN}

El cáncer de cuello uterino en etapa IB2 (tumor confinado al cuello uterino mayor de $4 \mathrm{~cm}$ ) continúa siendo un desafío en el manejo diario de nuestra subespecialidad. Sabemos que la sobrevida global ha aumentado en cáncer de cuello uterino etapa IB2 debido a la incorporación de la quimioterapia (1). Sin embargo, a pesar que la radioterapia con quimioterapia exclusiva concomitante sigue siendo el estándar de tratamiento, han surgido distintas alternativas de manejo en la búsqueda de una mejora en la sobrevida y en la calidad de vida de estas pacientes.

El realizar histerectomía post-radioterapia nació en 1969 con Durrance y cols (2), quienes reportan que la incidencia de recurrencia local podría ser reducida de $15 \%$ a $2,6 \%$ con la incorporación de la cirugía. La geometría de estos grandes tumores cervicales y la hipoxia asociada sería mejor tratada con la histerectomía mas que el agregar braquiterapia. Perez y cols (3) y Thoms y cols (4) en los años 90 muestran en estudios retrospectivos que la sobrevida no es significativamente mejor al adicionar la cirugía después de la radioterapia. El único trabajo que evalúa la histerectomía extrafascial post-radioterapia en forma aleatorizada prospectiva es el GOG 71 (5), el cual muestra una menor recurrencia local en las pacientes en las cuales se realiza la cirugía.

La quimioterapia neoadyuvante y la cirugía es otra alternativa de tratamiento investigada en estos casos, existiendo diferencias en los resultados y conclusiones que no permiten adoptar esta conducta como una alternativa de manejo estándar (6-7).

El objetivo principal de este trabajo es evaluar la sobrevida de pacientes con cáncer de cuello uterino etapa IB2, tratados con histerectomía extrafascial posterior a radioquimioterapia, y como objetivo secundario comparar la sobrevida entre subgrupos según la presencia o no de enfermedad residual en la pieza operatoria.

\section{PACIENTES Y MÉTODO}

Esta es una serie de casos de pacientes con cáncer cervicouterino etapa IB2, tratados con radio- quimioterapia y posterior histerectomía extrafascial en los Hospitales Gustavo Fricke de Viña del Mar y Carlos Van Buren de Valparaíso, entre enero de 2005 y diciembre de 2010.

El tratamiento consistió en radioterapia pelviana externa y un implante de braquiterapia úterovaginal con dosis preoperatoria entre 70 y 75 Gy al punto $\mathrm{A}$, concomitante a una dosis de Cisplatino $40 \mathrm{mg} / \mathrm{m} 2$ semanal por cinco semanas, seguido de histerectomía extrafascial, programada entre 4 y 6 semanas después de terminada la radioquimioterapia. Los datos fueron recolectados mediante la revisión retrospectiva de las fichas clínicas de las pacientes registradas en las bases de datos de las Unidades de Ginecología Oncológica de cada hospital.

Los criterios de inclusión fueron: pacientes con diagnóstico histológico de cáncer cervicouterino en etapa IB2, determinada clínicamente mediante examen ginecológico, y que fueron sometidas a histerectomía extrafascial posterior al tratamiento con radioquimioterapia. No hubo criterios de exclusión.

Para el análisis estadístico se utilizó el programa Stata 11.0, estimando la sobrevida mediante curvas de Kaplan Meier. La comparación de la sobrevida entre subgrupos se realizó utilizando la dócima de log-rank, con un $\alpha=0,05$.

\section{RESULTADOS}

Se reclutaron 31 pacientes con cáncer cervicouterino en etapa IB2. El promedio de edad fue de $41 \pm 8$ años. En la histología se encontró carcinoma escamocelular en 25 pacientes $(81 \%)$ y adenocarcinoma en 6 (19\%). El tamaño inicial del cuello uterino fue en promedio de $5,3 \pm 0,8 \mathrm{~cm}$ y posterior a la radioquimioterapia $3 \pm 0,9 \mathrm{~cm}(\mathrm{p}<0,0001$ para la prueba de t pareada, verificando la distribución normal de la variable mediante la prueba de ShapiroWilk).

Tres pacientes presentaron ganglios pelvianos sospechosos en la Tomografía Axial Computada Pelviana, y de éstas, una presentó recurrencia a distancia posterior al tratamiento por compromiso lumboaórtico. Recibieron 5 ciclos de quimioterapia 23 pacientes $(79 \%)$ y $4(14 \%)$ cuatro ciclos. El $7 \%$ restante corresponde a una paciente que recibió 3 
ciclos y otra un ciclo solamente por presentar reacciones adversas. La duración de la radioterapia fue en promedio de $53,4 \pm 9,5$ días (rango: 40 y 77 días). La histerectomía extrafascial se realizó 4 a 6 semanas post-radiación en el $85 \%$ de los casos. No hubo complicaciones atribuibles a la cirugía.

En 23 pacientes (74\%) no se encontró enfermedad residual en la pieza operatoria. En 7 (22\%) se encontró enfermedad residual microscópica y enfermedad macroscópica en 1. Cuatro pacientes (13\%) presentaron recurrencia locorregional posterior al tratamiento. Una paciente presentó recurrencia a distancia y otra recidivó a nivel locorregional y a distancia. La mortalidad de esta serie fue de $9,73 \%$ (3 pacientes), todas atribuibles a progresión de su enfermedad.

La mediana de seguimiento fue de 38 meses. La sobrevida global y libre de enfermedad estimada a 5 años fue de $86 \%$ y $79 \%$ respectivamente (Figuras 1 y 2). Hubo diferencia estadísticamente significativa en la sobrevida global entre los subgrupos sin enfermedad residual y con enfermedad residual macroscópica en la pieza operatoria $(p<0,001$ para la prueba de log rank). Lo mismo ocurre al comparar los grupos con enfermedad residual microscópica y macroscópica ( $p=0,0143$ para la prueba de log rank) (Figura 3). Sin embargo, al comparar la sobrevida del grupo de pacientes con enfermedad residual microscópica solamente versus las pacientes sin enfermedad residual, la diferencia no es significativa ( $p=0,47$ para la prueba de log rank). La única paciente con enfermedad residual macroscópica falleció a los 17 meses de seguimiento (Figura 3).

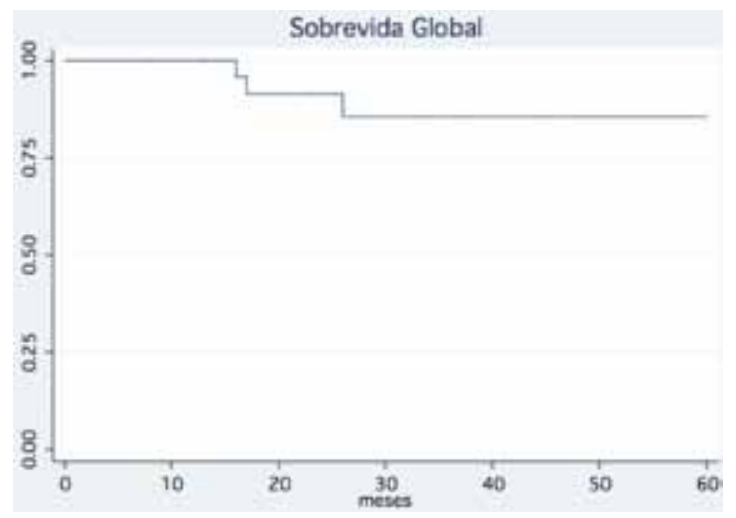

Figura 1. Curva de Kaplan-Meier estimando sobrevida global para pacientes con cáncer de cuello uterino etapa IB2 tratadas con histerectomía extrafascial posterior a radioquimioterapia: $86 \%$ a 5 años.

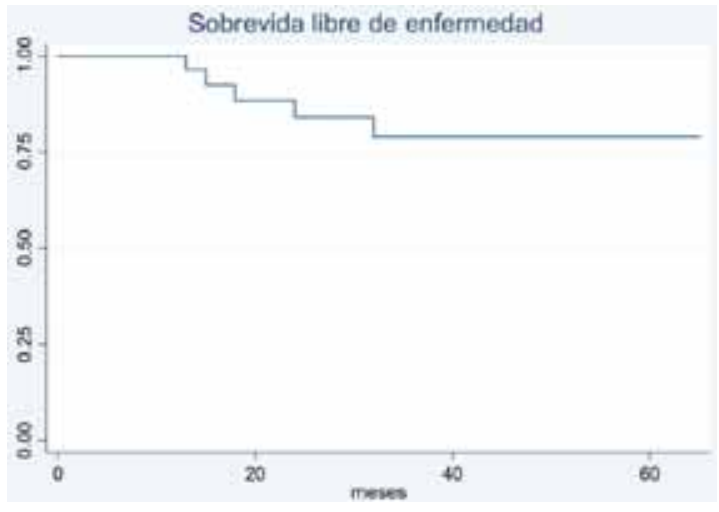

Figura 2. Curva de Kaplan-Meier estimando sobrevida libre de enfermedad para pacientes con cáncer de cuello uterino etapa IB2 tratadas con histerectomía extrafascial posterior a radioquimioterapia: $79 \%$ a 5 años.

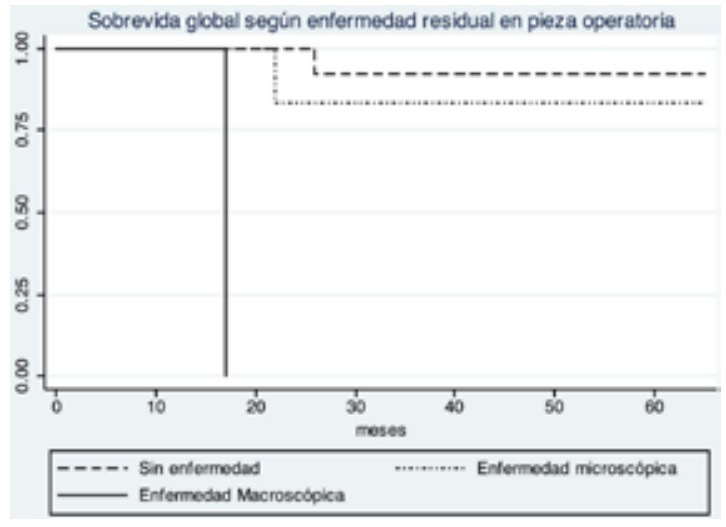

Figura 3. Curvas de Kaplan-Meier estimando sobrevida libre de enfermedad para pacientes con cáncer de cuello uterino etapa IB2 tratadas con histerectomía extrafascial posterior a radioquimioterapia según enfermedad residual en la pieza operatoria. Se compararon las sobrevidas con el test de log rank. Existió una diferencia estadísticamente significativa al comparar el grupo con enfermedad residual macroscópica y los otros dos grupos, pero no hubo diferencias al comparar el grupo con enfermedad residual microscópica y sin enfermedad residual.

\section{DISCUSIÓN}

La Federación Internacional de Ginecología Oncológica (FIGO) reportó el 2006 una sobrevida global del $75,6 \%$ a 5 años en el grupo de pacientes con cáncer de cuello uterino en etapa IB2 (8). De los trabajos que evalúan la sobrevida global en 
este mismo grupo de pacientes la mayoría son retrospectivos y sólo algunos incluyen el uso de quimioterapia concomitante. No existen estudios que evalúen exclusivamente la histerectomía extrafascial posterior a radioquimioterapia y toda la información con que contamos en la actualidad se refiere exclusivamente a estudios retrospectivos y a la inferencia que podemos hacer de dos estudios prospectivos y aleatorizados: el GOG 71 y el GOG 123.

El GOG 71 es el único trabajo que evalúa la histerectomía extrafascial post-radioterapia (sin quimioterapia) en forma aleatorizada prospectiva en el cáncer de cuello uterino etapa IB2 (5). En La recurrencia local tuvo una incidencia menor en el grupo con histerectomía ( $27 \%$ vs $14 \%$ a 5 años) y las conclusiones son ambiguas, sugiriendo que no existiría un beneficio clínico importante al agregar la histerectomía (sobrevida global a 5 años de $56 \%$ vs $58 \%$ con y sin histerectomía respectivamente), pero que hay una buena evidencia para pensar que pacientes con tamaños tumorales entre 4,5 y $6 \mathrm{~cm}$ se beneficiarían de la cirugía. Al evaluar si existen diferencias según el compromiso de la pieza operatoria, se encontraron diferencias significativas en la sobrevida libre de enfermedad y la sobrevida global entre las pacientes sin tumor residual y con tumor residual (7 veces más riesgo de morir en presencia de tumor macroscópico comparado con la ausencia de tumor). EI GOG 123 tiene como objetivo evaluar la quimioterapia concomitante en el grupo de pacientes con cáncer de cuello etapa IB2, pero en ambos grupos aleatorizados se realizó la histerectomía extrafascial posterior a la radioquimioterapia, por lo que sólo podemos destacar el resultado principal de sobrevida global y libre de enfermedad a 3 años de $83 \%$ y $79 \%$ respectivamente en ese grupo de pacientes. Estos serían similares también a las sobrevidas observadas en esta serie de casos.

En trabajos retrospectivos similares a este, Classe et al (9) el 2006 reportan una sobrevida global a 5 años de $78,9 \%$ en las pacientes con cáncer de cuello uterino IB2 tratadas con histerectomía radical posterior a radioquimioterapia. En Chile, San Martin y cols en el 2003 (10), publicaron una experiencia con 63 pacientes tratadas de la misma forma que las reportadas en este manuscrito, obteniendo una sobrevida global estimada a 5 años de $88 \%$, similar a la nuestra (86\%).

Según la evidencia existente es difícil definir si existe un claro beneficio al agregar la histerectomía extrafascial en pacientes con cáncer cervicouterino en etapa IB2. Sin embargo, la cirugía (histerectomía radical o extrafascial) después de radioquimioterapia no ha sido aún evaluada en estudios fase III.

Existen estudios recientes que describen la cirugía posterior al tratamiento de radioterapia más quimioterapia concomitante como una alternativa para evaluar la respuesta patológica al tratamiento y mejorar el control local en quienes tienen una respuesta parcial $(9,11)$. En este trabajo el análisis de subgrupos según enfermedad residual en la pieza operatoria de la histerectomía, el con enfermedad residual macroscópica tuvo una sobrevida significativamente menor que los con presencia de enfermedad residual microscópica o ausencia de enfermedad. Sin embargo, esta serie cuenta con sólo una paciente con enfermedad residual macroscópica. El hecho de que las curvas de sobrevida entre los grupos con ausencia de tumor y enfermedad microscópica en la pieza operatoria no muestren una diferencia significativa como en el GOG 71 (5), puede estar dado fundamentalmente por el tamaño muestral. Es importante destacar que la paciente con enfermedad residual macroscópica completó adecuadamente su tratamiento, tanto en el tiempo de radioterapia como en los ciclos de quimioterapia recibidos, mientras que dos pacientes con enfermedad residual microscópica no recibieron el tratamiento completo. Una recibió sólo 4 ciclos de quimioterapia, realizándose a las 3 semanas la histerectomía, y otra paciente no completó el tratamiento de radioterapia en los 55 días esperados. De estos resultados podemos hipotetizar que la presencia de tumor residual macroscópico en la pieza operatoria podría ser un factor de valor pronóstico.

En nuestra casuística no se registraron complicaciones derivadas de la histerectomía, por lo que en esta serie, el adicionar cirugía no constituyó un factor que limite su aplicación. En la serie del GOG 71 (5), tampoco se reportan diferencias en las complicaciones grado 3 y 4 que pudieran ser atribuidas a la cirugía.

\section{CONCLUSIÓN}

Esta serie muestra resultados de sobrevida similares a las reportadas en otros trabajos para el mismo grupo de pacientes con radioquimioterapia e histerectomía posterior, sin agregar complicaciones con la cirugía. La presencia de enfermedad residual en la pieza operatoria podría constituir un factor de valor pronóstico. La validación de este manejo requiere de ensayos clínicos con tamaños muestrales adecuados. 


\section{REFERENCIAS}

1. Keys HM, Bundy BN, Stehman FB, Muderspach LI, Chafe EW, et al. Cisplatin, radiation, and adjuvant hysterectomy compared with radiation and adjuvant hysterectomy for bulky satge IB cervical carcinoma. N Engl J Med 1999;340:1154-61.

2. Durrance FY, Fletcher GH, Rutledge FN. Analysis of central recurrent disease in stage I and II squamous cell carcinomas of the cervix on intact uterus. Am $\mathrm{J}$ Roentgenol Rad Ther Nuclear Med 1969;106:831-8.

3. Perez CA, Grigsby PW, Camel HM, Galakatos AE, Mutch D, Lockett MA. Irradiation alone or combined with surgery in stage IB, IIA and IIB carcinoma of uterine cervix: update of a nonrandomized comparison. Int J Radiat Oncol Biol Phys 1995;31:703-16.

4. Thoms WW Jr, Eifel PJ, Morris M, Delclos L, Wharton JT, Oswald MJ. Bulky endocerviacl carcinoma: a 23 year experience. Int J Radiat Oncol Biol Phys 1992;23:491-9.

5. Keys HM, Bundy BN, Stehman FB, Okagaki T, Gallup DG, Burnett AF, et al. For the Gynecology Oncology Group. Radiation therapy with and without extrafascial hysterectomy for bulky stage IB cervical carcinoma: a randomized trial of the Gynecologic Oncology Group. Gynecol Oncol 2003;89:343-53.

6. Chen $\mathrm{H}$, Liang $\mathrm{C}$, Zhang L, Huang $\mathrm{S}$, Wu X. Clinical efficacy of modified preoperative neoadjuvant chemotherapy in the treatment of locally advanced (stage IB2 to IIB) cervical cancer: randomized study. Gynecol Oncol 2008;110:308-15.
7. Eddy GL, Bundy BN, Creasman WT, Spirtos NM, Mannel RS, Hannigan E, O'Connor D. Treatment of ("bulky") stage IB cervical cancer with or without neoadjuvant vincristine and cisplatin prior to radical hysterectomy and pelvic/para-aortic lymphadenectomy: a phase III trial of the gynecologic oncology group. Gynecol Oncol 2007;106:362-9.

8. Quinn MA, Benedet J, Odicino F, et al. Carcinoma of the cervix uteri: annual report on the results of treatment in gynecological cancer. Int J Gynecol Obstet 2006;95:543-5103.

9. Classe JM, Rauch P, Rodier JF, Morice P, Stoeckle E, Lasry S, Houvenaeghel G; Groupe des Chirurgiens de Centre de Lutte Contre le Cancer.Surgery after concurrent chemoradiotherapy and brachytherapy for the treatment of advanced cervical cancer: morbidity and outcome: results of a multicenter study of the GCCLCC (Groupe des Chirurgiens de Centre de Lutte Contre le Cancer). Gynecol Oncol 2006;102:523-9.

10. San Martín $S$, Suárez E, Olfos $P$, Escudero $P$, Rojas $L$, Larraguibel $R$, et al. Cáncer cervicouterino en estadio IB2: tratamiento con radioquimioterapia concomitante y cirugía. Rev Chil Obstet Ginecol 2003;68:447-57.

11. Motton S, Houvenaeghel G, Delannes M, Querleu D, Soulé-Tholy $M$, Hoff $J$, Lèguevaque $P$. Results of surgery after concurrent chemoradiotherapy in advanced cervical cancer: comparison of extended hysterectomy and extrafascial hysterectomy. Int J Gynecol Cancer 2010;20:268-75. 